\title{
pH EFFECT ON THE SYNTHESIS OF MAGNETITE NANOPARTICLES BY THE CHEMICAL REDUCTION- PRECIPITATION METHOD
}

\author{
Ângela L. Andrade*, Diana M. Souza, Márcio C. Pereira, José D. Fabris e Rosana Z. Domingues \\ Departamento de Química, Instituto de Ciências Exatas, Universidade Federal de Minas Gerais, CP 702, 31270-901 Belo Horizonte \\ - MG, Brasil
}

Recebido em 15/1/09; aceito em 9/9/09; publicado na web em 22/2/10

\begin{abstract}
This work aimed at putting in evidence the influence of the $\mathrm{pH}$ on the chemical nature and properties of the synthesized magnetic nanocomposites. Saturation magnetization measurements evidenced a marked difference of the magnetic behavior of samples, depending on the final $\mathrm{pH}$ of the solution after reaction. Magnetite and maghemite in different proportions were the main magnetic iron oxides actually identified. Synthesis with final $\mathrm{pH}$ between 9.7-10.6 produced nearly pure magnetite with little or no other associated iron oxide. Under other synthetic conditions, goethite also appears in proportions that depended upon the $\mathrm{pH}$ of the synthesis medium.
\end{abstract}

Keywords: magnetic nano-composites; magnetite; Mössbauer spectroscopy.

\section{INTRODUCTION}

Nanotechnology is a field of growing interest for many physicists and chemists, because the reduced dimension of the solid system leads to peculiar physical and chemical properties that differ from the corresponding bulk materials. The origin of these distinguished properties is either the large surface-to-volume ratio in the particles or the quantum-mechanical nature of electronic structure, which imposes size-dependent modulations or modifications on the chemical behavior, when the size of the particles reaches dimensions of the wavelength of valence electrons in the solid. ${ }^{1}$

A main driving force for nanoparticle research is the large potential on technological applications. In the case of magnetic nanostructures, applications include data storage technology, ${ }^{2}$ medical diagnostics as contrast enhancers in magnetic resonance imaging ${ }^{3}$ and drug deliverer. ${ }^{4}$

Among the iron oxides, magnetite $\left(\mathrm{Fe}_{3} \mathrm{O}_{4}\right)$ is a very promising candidate for biological applications for its biocompatibility ${ }^{5}$ and suitable magnetic properties. ${ }^{6}$ Biomedical applications also require that iron oxide nanoparticles should be discrete and superparamagnetic with uniformly small particle sizes distribution. ${ }^{7-9}$

Reported methods for the synthesis of magnetite nanoparticles include reduction of hematite with $\mathrm{CO} / \mathrm{CO}_{2}{ }^{10}$ or $\mathrm{H}_{2},{ }^{11}$ co-precipitation from the solution of ferrous/ferric mixed salt solution in alkaline medium followed by aging and digestion in the temperature range of $90-150{ }^{\circ} \mathrm{C} ; ;^{12}$ microwave hydrothermal ${ }^{13}$ and electrical discharge. ${ }^{14} \mathrm{Qu}$ et al. ${ }^{15}$ reported a method for the direct preparation of spherically-shaped magnetite nanoparticles smaller than $10 \mathrm{~nm}$, from aqueous solution of a ferric salt, which is stable in air. In such a method, the ferrous ions are formed by partial reduction of ferric ions with $\mathrm{Na}_{2} \mathrm{SO}_{3}$ before the precipitation agent is added.

In this work, it was studied the influence of the $\mathrm{pH}$, on the chemical nature and properties of magnetic nanocomposites, prepared by precipitation from partially reduced ferric chloride aqueous solutions, via a modified route from that originally proposed by Qu et al. ${ }^{15}$ The chemical and magnetic properties of the formed nanomaterials were investigated in detail.

\footnotetext{
*e-mail: angelala01@ @otmail.com
}

\section{EXPERIMENTAL}

\section{Materials}

Ferric chloride hexahydrate, ammonium hydroxide, sodium sulfite anhydrous (Vetec, 30\%), and hydrochloric acid (Synth) reagent grade chemicals were used in this synthesis.

\section{Synthesis}

Essentially, it was used the method described by $\mathrm{Qu}$ et al. ${ }^{15}$ $20 \mathrm{~mL}$ of $1 \mathrm{~mol} \mathrm{~L}^{-1} \mathrm{Na}_{2} \mathrm{SO}_{3}$ stock solution was added to $30 \mathrm{~mL}$ of $2 \mathrm{~mol} \mathrm{~L} \mathrm{LeCl}_{3} \cdot 6 \mathrm{H}_{2} \mathrm{O}$ stock solution, previously acidified with 3, 2, $1,0.5$ and $0.25 \mathrm{~mol} \mathrm{~L}^{-1} \mathrm{HCl}$ solution. The reaction was carried out in a $1000 \mathrm{~mL}$ 3-necked round bottom flask by bubbling-in a protective $\mathrm{N}_{2}$ gas to assure an inert atmosphere. After mixing $\mathrm{Fe}^{3+}$ and $\mathrm{SO}_{3}{ }^{2-}$, the color of the solution changed from light yellow to red-brown and after few minutes, the yellow color reappeared. In this time, $750 \mathrm{~mL}$ of $0.5 \mathrm{~mol} \mathrm{~L}^{-1}$ ammonium hydroxide solution was then quickly poured into the solution under vigorous stirring; then a black precipitate was formed. Final $\mathrm{pH}$ of the solution after reaction was 8, 8.5, 9.7, 10.2 and 10.6 for samples previously acidified with $3,2,1,0.5$ and $0.25 \mathrm{~mol} \mathrm{~L}^{-1} \mathrm{HCl}$ solution, respectively. The reaction was continued by stirring for an additional $30 \mathrm{~min}$. The supernatant was discarded and the black precipitate was washed and centrifuged in distilled water. This procedure was repeated five times and the precipitate so obtained was centrifuged in an acetone medium and subsequently placed in a desiccator with silica beads to dry at room temperature.

The samples were labeled such as Mt8, Mt8.5, Mt9.7, Mt10.2 and Mt10.6, according to the $\mathrm{pH}$ of the solution after reaction. All samples were kept under vacuum, to avoid long-term oxidation by air.

\section{Characterization methods}

Total $\mathrm{Fe}$ was determined by volumetric titration with $\mathrm{K}_{2} \mathrm{Cr}_{2} \mathrm{O}_{7} \cdot{ }^{16}$ Chemical structure and homogeneity were evaluated from the FTIR spectra obtained in a Perkin Elmer Spectrum GX spectrophotometer. For these analyses, samples were compacted with $\mathrm{KBr}$ (approximately 1 mass \%) and analyzed in transmission mode.

Saturation magnetization was measured with a portable magnetometer, under a fixed magnetic field of $0.3 \mathrm{~T}$; the equipment was 
calibrated with metallic nickel. ${ }^{17}$ The powder X-ray diffraction (XRD) data were obtained in a Rigaku model Geigerflex, using $\mathrm{CuK} \alpha$ radiation scanning from 20 to $70^{\circ}$ at a scan rate of $2^{\circ} \mathrm{min}^{-1}$; silicon was used as external standard. The transmission Mössbauer spectra were collected at $298 \mathrm{~K}$ and $110 \mathrm{~K}$ in a constant acceleration transmission mode with a $\sim 30 \mathrm{mCi} \mathrm{Co}{ }^{57} / \mathrm{Rh}$ gamma-ray source. The spectrometer set up consisted of a transducer (CMTE model MA250) controlled by a linear function driving unit (CMTE model MR351).

\section{RESULTS AND DISCUSSION}

Colors of iron oxide are very dependent on grain size and chemical composition and this feature is a first auxiliary attribute in their identification, and may even be eventually used as a rule of thumb guide to purity. ${ }^{5,18}$ The Mt10.6, Mt10.2 and Mt9.7 samples were jet black and therefore suggesting being pure magnetite. The other samples, Mt8.5 and Mt8, generated brownish-red products, indicating a mixture of iron oxides. FT-IR, XRD, Mössbauer spectroscopy and saturation magnetization measurements were used to differentiate these products.

The chemical compositions for these magnetites, obtained by conventional chemical analyses (Table 1), have shown that the $\mathrm{Fe}^{2+}$ content is below the stoichiometric proportion for pure magnetite, particularly the Mt10.2 sample. $\mathrm{Fe}^{2+}$ was not detected in Mt8 sample, suggesting the absence of magnetite in that sample.

Table 1. Saturation magnetization measurements (s), lattice parameters and chemical formulae, deduced from iron chemical analysis and Mössbauer spectroscopy, for the Mt10.6, Mt10.2, Mt9.7, Mt8.5 and Mt8 samples

\begin{tabular}{|c|c|c|c|}
\hline Sample & $\sigma / \mathrm{J} \mathrm{kg}^{-1} \mathrm{~T}^{-1}$ & Lattice parameters $/ \AA$ & $\begin{array}{c}\text { Chemical Formula for } \\
\text { magnetite }\end{array}$ \\
\hline Mt10.6 & 60 & $8.383(7)$ & $\mathrm{Fe}_{2.11}^{3+} \mathrm{Fe}_{0.83}^{2+} \oplus_{0.06} \mathrm{O}_{4}$ \\
\hline Mt10.2 & 58 & $8.354(2)$ & $\mathrm{Fe}_{2.30}^{3+} \mathrm{Fe}_{0.55}^{2+} \oplus_{0.15} \mathrm{O}_{4}$ \\
\hline Mt9.7 & 60 & 8.3707 & $\mathrm{Fe}_{2.19}^{3+} \mathrm{Fe}_{0.72}^{2+} \oplus_{0.09} \mathrm{O}_{4}$ \\
\hline Mt8.5 & 31 & $\begin{array}{c}\text { Magnetite- } \mathrm{a}=8.386(3) \\
\text { Maghemite- } \mathrm{a}=8.34(3) \\
\text { Goethite }(\mathrm{a}=4.615(5) \\
\mathrm{b}=9.96(1) \text { and } \mathrm{c}=3.034(3)\end{array}$ & $\mathrm{Fe}_{2.20}^{3+} \mathrm{Fe}_{0.70}^{2+} \oplus_{010} \mathrm{O}_{4}$ \\
\hline Mt8 & 10 & $\begin{array}{c}\text { Maghemite- } \mathrm{a}=8.342(1) \\
\text { Goethite- } \mathrm{a}=4.585(3) \\
\mathrm{b}=9.911(5) \text { and } \mathrm{c}=3.0207(9)\end{array}$ & - \\
\hline
\end{tabular}

$\oplus$, cationic vacancy.

Figure 1 shows FTIR transmittance spectra of typical samples. The transmittance band at 582 and the $430 \mathrm{~cm}^{-1}$, belonging to the stretch vibration mode and torsional vibration mode of $\mathrm{Fe}-\mathrm{O}$ bonds in the tetrahedral sites and in the octahedral sites. In comparison with the literature, ${ }^{19}$ these two IR peaks of our products are shifted to higher wavenumbers, this may be due to the ultrafine particle sizes. When the size of $\mathrm{Fe}_{3} \mathrm{O}_{4}$ particles is reduced to nanoscale dimensions the bond force constant increases, because a large number of bonds involving surface atoms are broken, resulting in a rearrangement of non-localized electrons on the particle surface. ${ }^{5}$ Therefore, the FTIR spectrum of $\mathrm{Fe}_{3} \mathrm{O}_{4}$ nanoparticles exhibits a blue shift and the characteristic absorption bands of the $\mathrm{Fe}-\mathrm{O}$ bond are shifted to high wavenumbers by about $590 \mathrm{~cm}^{-1}$ (Figure 1).

The FTIR for Mt10.6 sample (Figure 1a) presents a band at $590 \mathrm{~cm}^{-1}$ (in the magnetite transmission region), whereas spectra for Mt10.2, Mt9.7, Mt8.5 and Mt8 samples (Figure 1b-e), respectively, present a broad band between 590 and $650 \mathrm{~cm}^{-1}$, in the magnetite and maghemite transmission region. Spectra for Mt8.5 and Mt8 samples (Figure 1d, e) present also other bands at 900 and $800 \mathrm{~cm}^{-1}$, which are typical of goethite $(\alpha \mathrm{FeOOH}){ }^{5,18}$

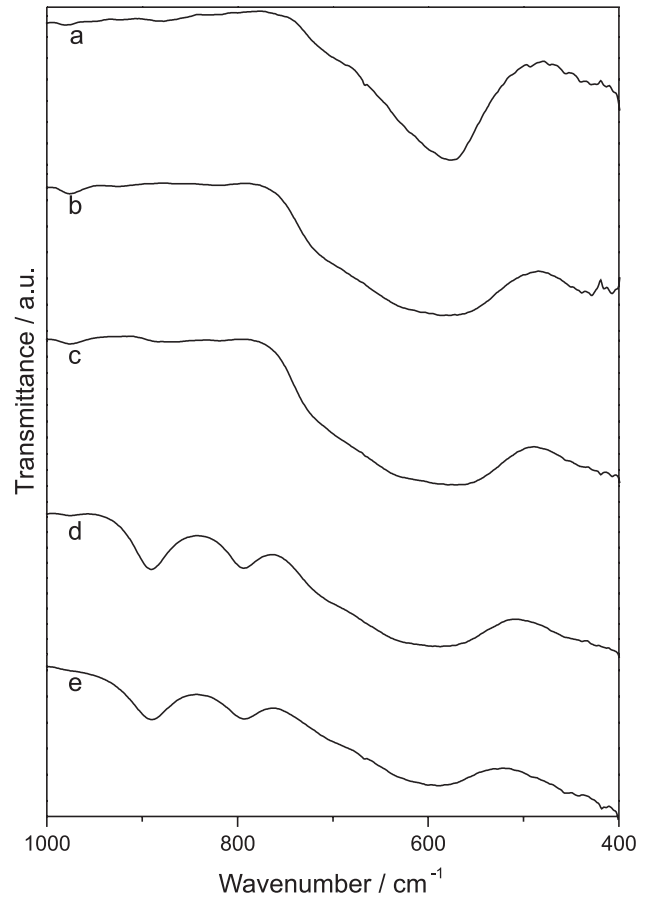

Figure 1. FTIR spectra of (a) Mt10.6, (b) Mt10.2, (c) Mt9.7, d) Mt8.5 e) Mt8 samples

The results obtained by XRD are shown in the Figure 2. X-ray diffraction patterns show characteristic symmetric reflections that suggest the presence of essentially a single crystallographic phase, corresponding to magnetite, with values of cubic lattice parameter $\mathrm{a}=8.383(7) ; 8.354(2)$ and $8.3707 \AA$ for Mt10.6, Mt10.2 and Mt9.7 samples, respectively (Table 1). Pattern for the sample Mt8.5 shows reflections corresponding to magnetite $(\mathrm{a}=8.386(3) \AA)$, one more oxidized specie, probably maghemite $(\mathrm{a}=8.34(3) \AA)$ and goethite $(\mathrm{a}=4.615(5), \mathrm{b}=9.96(1)$ and $\mathrm{c}=3.034(3) \AA)$. For Mt8, it was observed characteristic reflections of maghemite $(\mathrm{a}=8.342(1) \AA)$, and goethite $(\mathrm{a}=4.585(3), \mathrm{b}=9.911(5)$ and $\mathrm{c}=3.0207(9))$. Nano-sized magnetite is less stable than its bulk form and can be more readily oxidized to $\gamma \mathrm{Fe}_{2} \mathrm{O}_{3} \cdot{ }^{18}$

XRD patterns present line broadenings presumably due to small particle sizes. From reflection curve fittings, values of full width at half maximum of each reflection were used to estimate the mean coherent lengths these magnetites, using Scherrer equation, and a rough measure of the average crystallite size for all these magnetite samples is found to be about $9 \mathrm{~nm}$.

$298 \mathrm{~K}$ and $110 \mathrm{~K}$ Mössbauer spectra are showed in the Figure 3. $298 \mathrm{~K}$ Mössbauer spectrum for the stoichiometric magnetite bulk consists of two sextets, one of them corresponding to high spin $\mathrm{Fe}^{3+}$ on tetrahedral sites $\left(B_{h f}=49 \mathrm{~T}\right)$ and other to mixed valence $\mathrm{Fe}^{2.5+}$ on octahedral sites $\left(B_{h f}=46 \mathrm{~T}\right) .{ }^{5}$ Electron delocalization causes the nucleus to sense an averaged valence. In this work, $298 \mathrm{~K}$ Mössbauer spectra for the studied materials showed asymmetrically broadened lines due to particle sizes distribution. Moreover, it is interesting to observe a strong hyperfine field decreasing relative to magnetite bulk, due to superparamagnetic relaxation effects. Superparamagnetic relaxation can be described by the equation

$\tau=\tau_{0} e^{(K V / K T)}$

where $\tau$ is the relaxation time, $\tau_{0}$ a constant in the order of $10^{-10}$ $\mathrm{s}, \mathrm{K}$ is the anisotropy costant (a measure for the resistance of the particle to spin direction reversals), $\mathrm{V}$ is the particle volume, $\mathrm{k}$ 


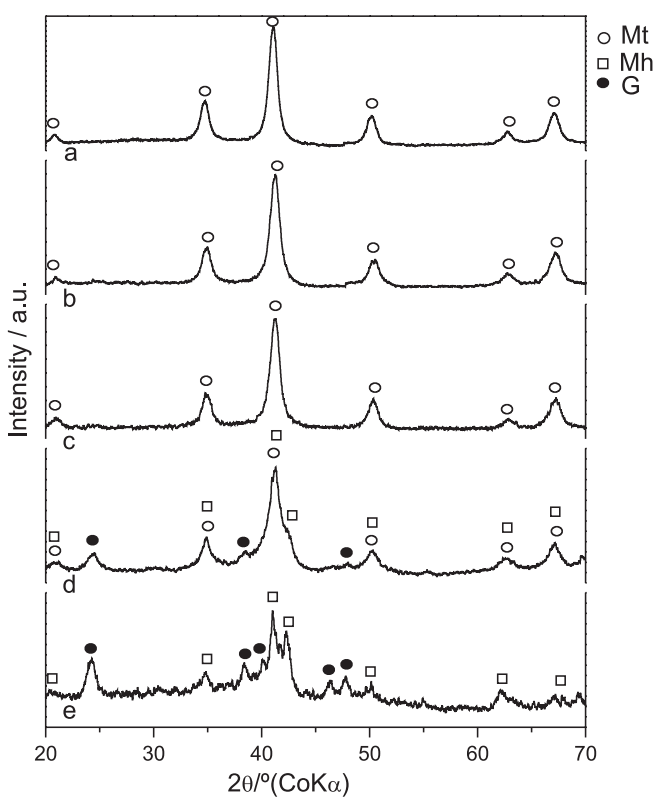

Figure 2. XRD spectra of (a) Mt10.6; (b) Mt10.2; (c) Mt9.7; (d) Mt8.5 and (e) Mt8samples. $M t=$ magnetite, $M h=$ maghemite, and $G=$ goethite phases

is the Boltzmann constant and $\mathrm{T}$ is the temperature. If the spin reversals occur more rapidly than nucleus can follow $\left(\tau_{\mathrm{L}} \approx 10^{-8} \mathrm{~s}\right.$ for ${ }^{57} \mathrm{Fe}$ ), the result will be a net reduction of the hyperfine field, down to zero for sufficiently small particles. Then, the reduction on the hyperfine field can expressed as:

$$
B_{h f}(\mathrm{~V}, \mathrm{~T})=B_{h f}(\mathrm{~V}=\infty, \mathrm{T})\langle\cos \theta\rangle
$$

in which $B_{h f}(\mathrm{~V}=\infty, \mathrm{T})$ is the hyperfine field in a large crystal at the same temperature and $\theta$ is the angle between the direction of magnetic moment and the easy direction of magnetization.

These results suggest that in addition to superparamagnetic relaxation, some collective excitation effects, reflecting small fluctuations in blocked particles, should also be considered..$^{20}$ Actually, superparamagnetic relaxation effects and collective magnetic excitation cause a significant line broadening and asymmetric lineshapes $;{ }^{20}$ resonance lines from octahedral and tetrahedral sites of magnetite do not clearly appear in the spectrum at room temperature, and this can explain the observed pattern for all samples in this study. Superparamagnetic goethite may explain the central doublets in Mt8.5 (Figure 3d) and Mt8 (Figure 3e) spectra. The maghemite identified in these samples by XRD do not clearly appear in the spectra at $110 \mathrm{~K}$ and room temperature. Mössbauer measurements with applied magnetic field are necessary to separate magnetite and maghemite in this case.

At $110 \mathrm{~K}$, the superparamagnetic relaxation effects practically disappear, except for the Mt8 (Figure 3e) that still presented a strong doublet due to goethite. Mt8.5 (Figure 3d) presented impurities of goethite $\left(B_{h f} \approx 48 \mathrm{~T}\right)$ and magnetite. Spectra for Mt10.6 (Figure 3a), Mt10.2 (Figure 3b) and Mt9.7 (Figure 3c) indicated only magnetite. The spectra of these samples also evidenced that this magnetite does not undergo Verwey transition at least down to $110 \mathrm{~K}$; the transition is very dependent on the structure, stoichiometry, impurity content, vacancies, stored stresses and particle sizes. $^{21}$

Saturation magnetization $(\sigma)$ values for magnetite bulk are around $100 \mathrm{~J} \mathrm{~T}^{-1} \mathrm{~kg}^{-1}{ }^{5,18}$ Magnetization values founded for Mt10.6, Mt10.2 and Mt9.7 are 60, 58 and $60 \mathrm{~J} \mathrm{~T}^{-1} \mathrm{~kg}^{-1}$, respectively. This decreasing
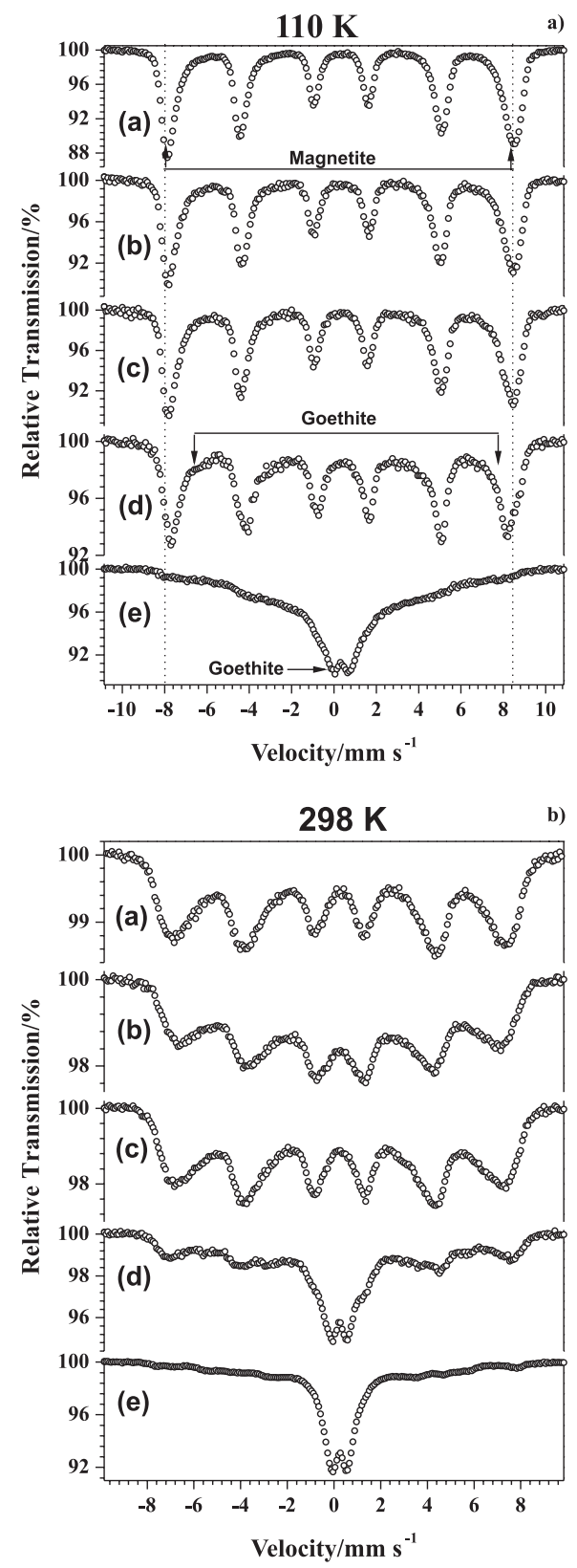

Figure 3. Mössbauer spectra for (a) Mt10.6; (b) Mt10.2; (c) Mt9.7 (d) Mt8.5 and (e) $M t 8$

of the magnetization value in respect to magnetite bulk is due to small particle sizes observed for these samples. For Mt8.5 and Mt8 samples, $\sigma$-values are 31 and $10 \mathrm{~J} \mathrm{~T}^{-1} \mathrm{~kg}^{-1}$, respectively. This low value of magnetization could be due to dilution of magnetite and maghemite on non-magnetic phases such as goethite.

These results suggest that optimal $\mathrm{pH}$ to produce pure magnetite nanoparticles must lie within the range 9.7 to 10.6 values. $\mathrm{pH}$ values below 8.5 lead to the formation of side-products to magnetite, specifically goethite and maghemite.

\section{CONCLUSIONS}

By combining results from Mössbauer spectroscopy, FTIR and $\mathrm{X}$-ray diffraction, it was possible to show a marked difference in the iron oxide composition of resulting magnetic particles, depending upon the synthesis condition, by varying the $\mathrm{pH}$. The material prepared within the $\mathrm{pH}$ values range 9.7 to 10.6 tends to contain pure 
magnetite with little or no other iron oxide. Other products, such as maghemite and goethite tend to appear when the synthesis is performed in $\mathrm{pH}$ values below 8.5.

\section{ACKNOWLEDGEMENTS}

The authors gratefully acknowledge the financial support of CNPq, FAPEMIG and CAPES (Brazil).

\section{REFERENCES}

1. Kalska, B.; Paggel, J. J.; Fumagalli, P.; Rybczynski, J.; Satula, D.; Hilgendorff, M.; Giersig, M.; J. Appl. Phys. 2004, 95, 1343.

2. Sun, S. H.; Murray, C. B.; J. Appl. Phys. 1999, 85, 4325 Part 2A.

3. Mitchell, D. G. J.; Magn. Reson. Imaging 1997, 7, 1.

4. Bonnemain, B.; J. Drug Target. 1998, 6, 167.

5. Schwertmann, U.; Cornell, R. M.; Iron oxides in the laboratory: preparation and characterization, Cambridge: New York, 1991.

6. Shinkai, M.; J. Biosci. Bioeng. 2002, 94, 606.

7. Pankhurst, Q. A.; Connolly, J.; Jones, S. K.; Dobson, J.; J. Phys., D Appl. Phys. 2003, 36, R167.

8. Tartaj, P.; Morales, M. D.; Veintemillas-Verdaguer, S.; GonzalezCarreno, T.; Serna, C. J.; J. Phys., D Appl. Phys. 2003, 36, R182.
9. Berry, C. C.; Curtis, A. S. G.; J. Phys., D Appl. Phys. 2003, 36, R198.

10. Darken, L. S.; Gurry, R. W.; J. Am. Chem. Soc. 1946, 68, 798.

11. Osterhout, V.; Magnetic Oxides, Wiley: New York, 1975.

12. Visalakshi, G.; Venkateswaran, G.; Kulshreshtha, S. K.; Moorthy, P. N.; Mater. Res. Bull. 1993, 28, 829.

13. Fan, R.; Chen, X. H.; Gui, Z.; Liu, L.; Chen, Z. Y.; Mater. Res. Bull. 2001, 36, 497 .

14. Wang, C. Y.; Zhu, G. M.; Chen, Z. Y.; Lin, Z. G.; Mater. Res. Bull. 2002, $37,2525$.

15. Qu, S. C.; Yang, H. B.; Ren, D. W.; Kan, S. H.; Zou, G. T.; Li, D. M.; Li, M. H.; J. Colloid Interface Sci. 1999, 215, 190.

16. Vogel, A.I.; A Text Book of Quantitative Inorganic Analysis, Longman: London, 1962.

17. Coey, J. M. D.; Cugat, O.; MaCauley, J.; Fabris, J. D.; Rev. Fis. Apl. Instrum. 1992, 7, 25.

18. Cornell, R. M.; The Iron Oxides: Structure, Properties, Reactions, Occurrences and Uses, Wiley: New York, 2003.

19. Nyquist, R. A.; Kagel, R. O.; Infrared Spectra of Inorganic Compounds, Academic Press: New York, London, 1971.

20. Morup, S.; Topsoe, H.; Lipka, J.; J Phys (Paris) 1976, 12, 287.

21. Bancroft, G. M.; Mössbauer spectroscopy: an introduction for in inorganic chemists and geochemists, MacGraw-Hill: London, 1973. 